\title{
Ultrastructure and Cell-Cell Coupling of Cardiac Myocytes Differentiating in Embryonic Stem Cell Cultures
}

\author{
Margaret V. Westfall,* Krystyna A. Pasyk, David I. Yule, Linda C. Samuelson, \\ and Joseph M. Metzger
}

Department of Physiology, University of Michigan, Ann Arbor, Michigan

\begin{abstract}
Differentiation cultures of embryonic stem (ES) cells can be a useful in vitro system for understanding cardiac myocyte development. However, cell morphometry, sarcomere development, and functional cell-cell junction formation have not been examined in detail to determine whether ES cell-derived cardiac myocytes exhibit structural and functional characteristics similar to cardiac myocytes within the developing heart. Therefore, we examined cellular dimensions, sarcomere formation, and cell-cell contacts in differentiating cardiac myocytes derived from mouse D3-ES cell cultures. Cells exhibited rod-shaped morphology and had single centrally located nuclei, typical of maturing cardiac myocytes. The cellular dimensions of 59 individual cardiac myocytes within contracting foci of ES cell cultures were analyzed (length $=42.2 \pm 2.1 \mu \mathrm{m}$, area $=197 \pm 19 \mu \mathrm{m}^{2}$, and diameter $=5.5 \pm 0.3 \mu \mathrm{m})$ and found to be similar to myocytes in vivo. Transmission electron micrographs of ES cell-derived cardiac myocytes indicated myofibrillar architecture ranged from sparse and disorganized to densely packed, parallel arrays of myofibrils organized into mature sarcomeres. This pattern of myofibrillar assembly in maturing sarcomeres was similar to that observed during in vivo myocyte differentiation. Another hallmark of cardiac development is the formation of intercalated discs, which functionally couple adjacent cardiac myocytes. Electron micrographs indicated nascent intercalated discs were forming in foci of ES cell-derived cardiac myocytes. In addition, indirect immunostaining with anti-connexin 43 antibody ( $\mathrm{Ab}$ ), a monoclonal $\mathrm{Ab}$ to the gap junction component of the intercalated disc, indicated that gap junctions were present in contracting ES cell foci. Furthermore, microinjection of single cardiac myocytes with Lucifer yellow $(2.5 \mu \mathrm{M})$ resulted in the spread of fluorescence to adjacent cells within a contracting focus, an indication of functional cell-cell coupling across these gap junctions. Together, these results indicate ES cell-derived cardiac myocytes exhibit cell morphology, sarcomere formation, and cell-cell junctions similar to those observed in cardiac myocytes developing in vivo. Cell Motil. Cytoskeleton 36:43-54, 1997. @ 1997 Wiley-Liss, Inc.
\end{abstract}

Key words: cardiogenesis; embryonic stem cells; sarcomere; morphometry; gap junctions; connexin 43

\section{INTRODUCTION}

Embryonic stem (ES) cells cultured as embryoid bodies (EBs) or in attached cultures have the capacity to differentiate in vitro into several cell types including cardiac myocytes [Doetschman et al., 1985; Metzger et al., 1994; Robbins et al., 1992]. In both EBs and attached ES cell cultures, foci of cardiac myocytes are observed
Contract grant sponsors: NIH, the American Heart Association, and the American Heart Association of Michigan.

*Correspondence to: Margaret V. Westfall, Dept. of Physiology, 7730 Med. Sci. II, 1301 E. Catherine St., University of Michigan, Ann Arbor, MI 48109-0622.

Received 16 May 1996; accepted 9 September 1996. 
and these foci contact spontaneously [Doetschman et al., 1985; Metzger et al., 1994]. Robbins et al. [1992] have suggested that ES cell-derived cardiac myocytes can be a useful model for understanding normal and abnormal in vivo cardiac myocyte development. This proposal is based in part, on studies of contractile protein gene expression in differentiating ES cell-derived cardiac myocytes.

Studies in complex aggregates of ES cells, known as EBs, also indicate that myosin heavy chain (MHC), tropomyosin and myosin light chain isoform expression patterns are consistent with the in vivo development program [Miller-Hance et al., 1993; Muthuchamy et al., 1993; Robbins et al., 1990]. However, multiple contracting foci within EBs have made it difficult to prove that the pattern of isoform expression is related to differentiation of a single contracting focus. We have studied contractile protein expression and the functional characteristics of differentiating cardiac myocytes in attached ES cell cultures [Metzger et al., 1994, 1995; Westfall et al., 1996]. The onset and duration (in days) of contraction are easily monitored in attached ES cell cultures [Metzger et al., 1994] and, thus, developmental changes within specific foci of myocytes can be correlated with the length of time that a focus contracts. The contractile protein isoforms and functional properties initially expressed in foci of contracting ES cell-derived cardiac myocytes are similar to those observed in myocytes from fetal/neonatal mice. Foci contracting for longer periods of time display contractile protein expression and functional characteristics similar to adult cardiac myocytes. More specifically, myofilament $\mathrm{Ca}^{2+}$ sensitivity decreases as foci contract for longer periods of time, as is the case during in vivo cardiogenesis [Metzger et al., 1994]. In addition, foci contracting for short intervals express slow skeletal muscle troponin I (ssTnI) [Westfall et al., 1996] and a greater proportion of $\beta$ - vs. $\alpha$-MHC [Metzger et al., 1995]. There is induction of cardiac TnI (cTnI) expression and an increase in $\alpha$-MHC expression in foci contracting for longer intervals. A transition from ssTnI and $\beta$-MHC expression to $\mathrm{cTnI}$ and $\alpha$-MHC expression is observed during rodent cardiogenesis in vivo [Saggin et al., 1989; Mahdavi et al., 1987]. These findings are consistent with foci of ES cell-derived cardiac myocytes undergoing a developmental transition from an embryonic/ fetal to an adult phenotype.

Collectively, these studies provide support for the idea that the ES cell-derived myocytes follow a similar developmental program as myocytes differentiating in vivo. The ability of ES cells to follow in vivo differentiation led Soonpaa et al. [1994] to suggest that ES cell-derived cardiac myocytes may provide a source of donor myocytes for myocardial repair. An essential first step prior to the use of these cells for intracardiac grafting is to determine whether cellular and ultrastructural organi- zation also follow a pattern similar to the in vivo program. However, the cell morphology and sarcomere ultrastructure of embryonic stem cell-derived cardiac myocytes during differentiation have not yet been analyzed in detail. Furthermore, no information is available on cellular junctions or functional coupling of these ES cell cardiac myocytes.

Therefore, in the present study, we characterized the cellular dimensions as well as sarcomeric and cellular junction ultrastructure of cardiac myocytes differentiating in attached embryonic stem cell cultures. Morphometric analysis of these cells indicate they are rod-shaped and span a range of sizes which encompass the dimensions observed in neonatal/fetal and adult cardiac myocytes. We also observed myofibrillar assembly and sarcomere formation during the course of ES cell cardiac myocyte differentiation. In addition, cell junctions were examined in the ES cultures. Nascent intercalated discs were observed, and within these junctions, we identified functional gap junctions specific for cardiac myocytes. Together, these results provide ultrastructural evidence consistent with previous myofibrillar protein expression and functional data showing that these cells follow the developmental program observed during cardiogenesis in vivo.

\section{MATERIALS AND METHODS Embryonic Stem Cell Cultures}

Attached differentiation cultures of mouse embryonic stem (ES) cells were prepared as described by Metzger et al. [1994, 1995]. Briefly, ES-D3 cells were cultured in DMEM on mitotically inactive mouse embryonic fibroblast (MEF) feeder cells supplemented with $15 \%$ fetal calf serum, $0.1 \mathrm{mM} \beta$-mercaptoethanol, and $2 \%$ of a leukemia inhibitory factor conditioned media to maintain the ES cells in the undifferentiated, pluripotential state [Metzger et al., 1994, 1995]. To initiate differentiation, ES cells were dispersed with trypsin and small aggregates were formed after seeding $~ 300$ ES cells in hanging drop suspension cultures. After incubating for 2 days, aggregates were transferred to suspension cultures for 3 days, during which time simple embryoid bodies (EBs) formed. Attached cultures were initiated by plating simple EBs onto gelatin-coated coverslips and incubating in DMEM media containing $20 \%$ fetal calf serum, 50 $\mathrm{U} / \mathrm{ml}$ penicillin, $50 \mu \mathrm{g} / \mathrm{ml}$ streptomycin sulfate, and 0.1 $\mathrm{mM} \beta$-mercaptoethanol [Metzger et al., 1994, 1995]. The onset and duration of contraction (in days) for each focus emerging in culture were then determined by monitoring cultures every other day for up to 18 weeks after attachment. 


\section{Preparation of Samples for Light and Transmission Electron Microscopy}

Isolation, fixation, and preparation of contracting foci of cardiac myocytes from ES cell cultures or adult mouse ventricles were carried out as described by Metzger et al. [1994]. Briefly, samples were fixed by immersion in Karnovsky's solution (3\% glutaraldehyde and 3\% formaldehyde in $0.1 \mathrm{M}$ sodium cacodylate buffer; $\mathrm{pH}$ 7.4) for 2 $\mathrm{h}$ at $4^{\circ} \mathrm{C}$. After fixation, samples were rinsed overnight with $7.5 \%$ saccharose in $0.1 \mathrm{M}$ sodium cacodylate buffer (pH 7.4) at $4^{\circ} \mathrm{C}$, post-fixed by immersion in $1 \%$ buffered osmium tetroxide for $1 \mathrm{~h}$ at room temperature, and dehydrated with graded concentrations of ethanol and propylene oxide. Next, they were incubated for $12 \mathrm{~h}$ in a 1:3 mixture of propylene oxide and epoxy resin, embedded in epoxy resin, and polymerized for 3 days at $45^{\circ} \mathrm{C}$ followed by 1 day at $60^{\circ} \mathrm{C}$.

For light microscopy, semi-thin sections $(\sim 600 \mathrm{~nm})$ were stained with toluidine blue, and morphometry was subsequently carried out using a Nikon Optiphot-2 microscope (Melville, NY) with a Hamamatsu C2400-50 CCD camera (Bridgewater, $\mathrm{NJ}$ ). In areas containing myocytes, live time images were passed through a media filter to obtain a more accurate boundary edge of the myocytes and then the contrast was adjusted to maximize the boundaries between myocytes. The resulting images were stored on an Image-1 system (Universal Imaging, West Chester, PA). For morphometry, the boundary of each myocyte was traced, and then the area, perimeter, longest chord (length), and maximum diameter of each myocyte was measured.

In preparation for electron microscopy, serial sections (50 nm thick) were cut with a diamond knife on a Sorvall MT-5000 ultramicrotome (RMC, Inc., Tucson, AZ). Ribbons of sections were mounted on copper grids, stained with aqueous uranyl acetate and lead citrate, and examined with a Philips CM-10 transmission electron microscope (Mehwah, NJ) at $60 \mathrm{kV}$.

\section{Indirect Immunofluorescence and Confocal Microscopy}

In preparation for immunocytochemistry, ES cell differentiation cultures were fixed for $30 \mathrm{~min}$ at room temperature with $3 \%$ paraformaldehyde in phosphate buffered saline (PBS, pH 7.40; $2.3 \mathrm{mM} \mathrm{NaH}_{2} \mathrm{PO}_{4}, 8.0$ $\mathrm{mM} \mathrm{Na}_{2} \mathrm{HPO}_{4}, 150 \mathrm{mM} \mathrm{NaCl}, 2.5 \mathrm{mM} \mathrm{KCl}$ ). Coverslips containing the cultures were rinsed in PBS to remove excess paraformaldehyde and then treated with $50 \mathrm{mM}$ $\mathrm{NH}_{4} \mathrm{Cl}$ for $30 \mathrm{~min}$ to remove unreacted aldehydes prior to immunostaining.

Indirect immunocytochemistry was then performed using a previously described dual monoclonal antibody (Ab) labeling protocol [Westfall et al., 1996]. Indirect immunofluorescence with dual monoclonal Abs was carried out with a cardiac specific anti-TnI Ab to identify cardiac myocytes followed by an $\mathrm{Ab}$ to the gap junction subunit, connexin $43(\mathrm{CX}-43)$ to determine whether gap junctions were present in these myocytes. Monoclonal $\mathrm{Ab}$ binding was detected with goat anti-mouse $\operatorname{IgG~} \mathrm{Ab}$ conjugated to either fluorescein isothiocyanate (FITC) or Texas Red (TR).

The specific protocol included an initial step to block nonspecific binding. Samples were incubated in PBS $+0.5 \%$ Triton X-100 (PBS-TX) and 20\% normal goat serum (NGS; Sigma, St. Louis, MO) for $30 \mathrm{~min}$ at room temperature. This step as well as all other blocking and immunolabeling steps were carried out in a humidity chamber. In all subsequent incubation steps, primary and conjugated secondary Abs were diluted in PBS-TX with $2 \%$ NGS and incubations were carried out at room temperature. Samples were then incubated for $1.5 \mathrm{~h}$ in TI-1 anti-cTnI monoclonal Ab (dilution = 1:5,000) [Saggin et al., 1989] (gift of S. Schiaffino) and rinsed in PBS-TX. Nonspecific secondary Ab binding was blocked with PBS-TX containing 2\% NGS, before incubation with TR-conjugated goat anti-mouse $\mathrm{Ab}$ (dilution = 1:100; Molecular Probes, Eugene, OR) for $1 \mathrm{~h}$. Unreacted sites on the TI-1 primary and TR-conjugated secondary Abs were neutralized by incubation in goat anti-mouse IgG (Sigma) overnight at $4^{\circ} \mathrm{C}$, and then in goat antimouse Fab fragments (Jackson Immunochemicals, West Grove, PA) for $1.5 \mathrm{~h}$ at room temperature. Both the $\mathrm{IgG}$ and Fab fragment were diluted 1:20 in PBS-TX with 2\% NGS. We have previously shown that these steps eliminated any cross-recognition by the second set of primary and secondary Abs [Westfall et al., 1996]. Samples were then blocked with $20 \%$ NGS in PBS-TX for $30 \mathrm{~min}$, incubated with monoclonal anti-CX-43 Ab (dilution = 1:1,000; Zymed, South San Francisco, CA) for $1.5 \mathrm{~h}$, and washed with PBS-TX. After a final 30 min blocking step with $2 \%$ NGS in PBS-TX, anti-CX-43 Ab binding was detected with a $1 \mathrm{~h}$ incubation with FITC-conjugated goat anti-mouse Ab (dilution = 1:200; Sigma). Samples were then rinsed in PBS and mounted as described by Westfall et al. [1996]. Immunolabeling was analyzed with a Bio-Rad (Richmond, CA) MRC 600 confocal microscope.

\section{Assessment of Functional Gap Junctions by Microinjection of Fluorescent Probes}

Contracting foci of cardiac cells within embryonic stem cell cultures were identified and then microinjected on the stage of an Attofluor imaging system (Rockville, MD), as described by Deutsch et al. [1995]. Briefly, pipettes were pulled with a micropipet forge (Sutter Instruments, Novato, CA) to a tip diameter $<0.1 \mu \mathrm{m}$ from $1.0 \mathrm{~mm}$ glass containing filament (SPI, Sarasota, FL). Pipettes were filled with $2.5 \mu \mathrm{M}$ Lucifer yellow and $5 \mathrm{mM}$ 


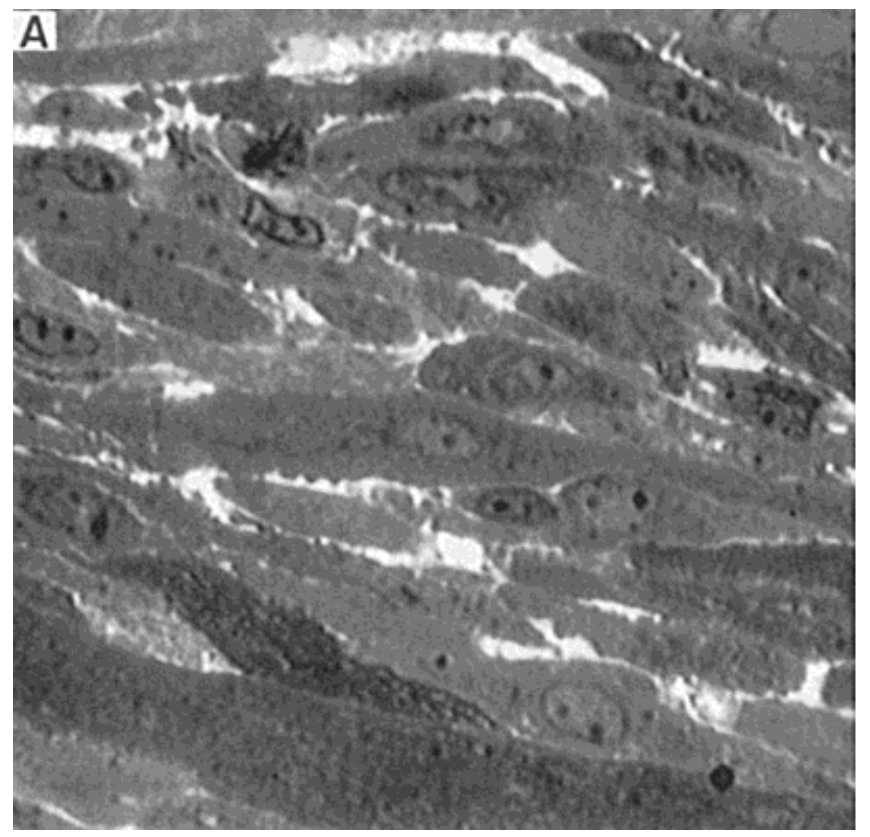

Fig. 1. Light micrograph (A) and representative Image-1 analysis of a single cardiac myocyte $(\mathbf{B})$ within a contracting focus of an attached ES cell culture. ES cultures were fixed, sectioned, and photographed as described in Methods. Maximum cardiac myocyte length, diameter,

TR which was coupled to dextran $(10 \mathrm{kDa})$, and then mounted in a perspex holder attached to a Burleigh piezo electric stepper (Fishers, NY). The final approach to and penetration of a cardiac myocyte within a focus of contraction was accomplished by making successive 2 $\mu \mathrm{m}$ steps until puncture occurred. Dye was injected with a pressure injector (Medical Systems Corporation, Greenvale, NY). After penetration of a single cell, and withdrawal of the pipette, the extent of dye injection was determined using an excitation $\lambda$ of $568 \mathrm{~nm}$ and emission $\lambda$ of $630 \mathrm{~nm}$ for TR-labeled dextran. Spread of Lucifer yellow signal was monitored with an excitation $\lambda$ of 488 $\mathrm{nm}$ and emission $\lambda$ of $530 \mathrm{~nm}$. The signal was monitored using an intensified CCD camera and videotape images were stored at various time points.

\section{RESULTS}

Analysis of the cellular dimensions and ultrastructure indicated that differentiating cardiac myocytes in ES cell culture and in vivo cardiac myocytes have similar properties. Cardiomyocytes within contracting foci of ES cell cultures (Fig. 1A) were overwhelmingly mononucleated and exhibited the rod-shaped morphology found in developing cardiac myocytes within the heart. Morphometric analysis was accomplished by measuring chord length, diameter, area, and the perimeter of individual ES cell-derived cardiac myocytes sectioned close to the

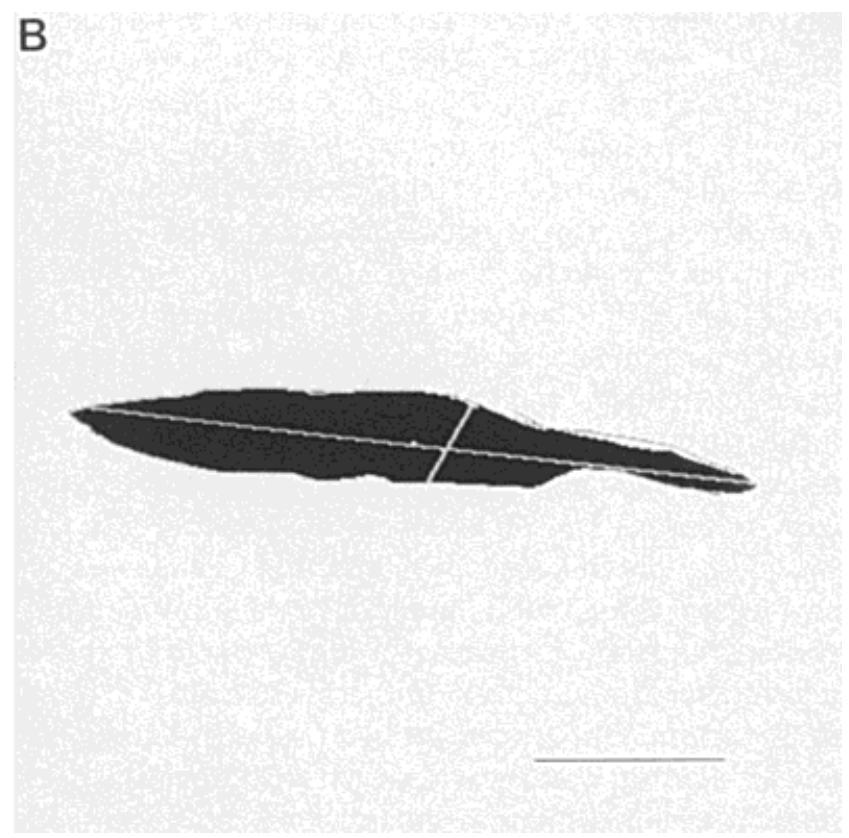

and perimeter were measured, and area was determined by tracing each cell with an Image-1 system. The representative cell shown in B was $71.2 \mu \mathrm{m}$ in length and $9.6 \mu \mathrm{m}$ in diameter. $\mathrm{Bar}=20 \mu \mathrm{m}$ and represents magnification in both $\mathrm{A}$ and $\mathrm{B}$.

center of the cell (Fig. 1B). Overall, the length, diameter, and area of ES cell cardiac myocytes (Table I) were within the range of dimensions previously published for neonatal $\left(\right.$ area $=44.8-61.9 \mu \mathrm{m}^{2}$, diameter $=7.5-8.5 \mu \mathrm{m}$, length $=21.7-44.7 \mu \mathrm{m}$ ) [Anversa et al., 1980] to adult rodent myocytes (length $=100-128 \mu \mathrm{m}$, diameter $=$ 20-25 $\mu \mathrm{m}$ [Korecky and Rakusan, 1978]; length = 80-150 $\mu \mathrm{m}$, diameter $=10-30 \mu \mathrm{m}$ [Severs, 1989]). However, the average length/diameter ratio was larger in ES cell-derived cardiac myocytes (Table I) compared to published values for neonatal ( $\mathrm{L} / \mathrm{D}=2.9$ ) [Anversa et al., 1980] and adult (L/D = 5.3) [Korecky and Rakusan, 1978] rodent ventricular myocytes. This result may reflect a difference between the factor(s) and/or signal(s) regulating cell size under in vivo vs. in vitro conditions.

Electron micrographs of individual ES cell cardiac myocytes were examined to determine whether myofibrillar architecture also was consistent with the pattern of sarcomere assembly observed during cardiac myocyte differentiation in vivo. Nascent myofibrils lacking alignment were observed in cells from precontractile foci or foci contracting for short durations (Fig. 2A,B). In some foci, $\mathrm{Z}$ bands also were forming (Fig. 2C). In Figure 2D, although still sparse, parallel bundles of myofibrils exhibited A and I bands. This range of myofibrillar architecture in foci contracting for short durations is consistent with the pattern of myofibrillar assembly observed during cardiac development in vivo [Bishop et al., 1990; Chacko, 
TABLE I. Morphometric Analysis of Rod-Shaped Cardiac Myocytes Within Contracting Foci of Attached ES Cell Cultures $(\mathbf{n}=59)$

\begin{tabular}{lcc}
\hline & Mean \pm SEM & Range \\
\hline Area $\left(\mu \mathrm{m}^{2}\right)$ & $197.2 \pm 18.9$ & $46.3-872.8$ \\
Perimeter $(\mu \mathrm{m})$ & $88.3 \pm 4.4$ & $57.6-211.4$ \\
Chord length $(\mu \mathrm{m})$ & $42.4 \pm 2.1$ & $27.0-103.1$ \\
Diameter $(\mu \mathrm{m})$ & $5.5 \pm 0.3$ & $2.4-13.1$ \\
Length/diameter ratio & $8.0 \pm 0.6$ & $3.0-19.3$ \\
\hline
\end{tabular}

1976; Manasek, 1970]. We were unable to assign these initial stages of myofibrillar assembly to a specific day of contraction because differentiation within a focus was not sufficiently uniform. Variations in myofibrillar assembly also occur among cells during the early stages of cardiac development in vivo [Chacko, 1976; Manasek, 1970].

To confirm that myofibrillar assembly was progressing in contracting foci of ES cell-derived cardiac myocytes, we examined myofibrillar architecture in a more mature focus of contraction (Fig. 3). This ES cell focus had been contracting for 36 days and was microdissected prior to fixation to eliminate the possibility that other foci contracting for shorter durations within the same culture were inadvertently examined. Densely packed, parallel bundles of myofibrils occupy much of the myocyte cell volume and sarcomeres have clearly delineated A and I bands along with $\mathrm{Z}$ discs in cardiac myocytes from the $\mathrm{ES}$ cell focus (Fig. 3A,B). This sarcomere architecture is more mature than the foci shown in Figure 2. Instead, sarcomeres in this focus were highly comparable to the architecture observed in adult mouse cardiomyocytes (Fig. 4). Adult myocytes also exhibited $\mathrm{H}$ and $\mathrm{M}$ bands (Fig. 4) which were faintly detected in some sarcomeres of ES cell-derived cardiac myocytes (Fig. 3A). Finally, mitochondria were packed adjacent to myofibrils/ sarcomeres in cardiac myocytes from adult mice (Fig. 4) as well as the ES cell focus contracting for 36 days (Fig. 3B).

In addition to myofibrillar architecture, we examined the developing sarcomere length in sections where myofibrillar maturation had progressed to the point where Z-bands were apparent (Figs. 2C,D, 3). In Figure 2C and $\mathrm{D}$, the developing sarcomere lengths were 2.67 and 2.70 $\mu \mathrm{m}$, respectively. Sarcomere length also was examined in several additional sections with a similar degree of myofibrillar assembly. The average sarcomere length of all sections in this group $(2.25 \pm 0.10 \mu \mathrm{m} ; \mathrm{n}=10)$ was slightly shorter than the sarcomere lengths in Figure 2. However, average sarcomere length was still longer than the sarcomere length observed in neonatal cardiomyocytes (1.81-1.94 $\mu \mathrm{m})$ [Anversa et al., 1980]. Differences in the pressure load and/or culture conditions surrounding the contracting foci may result in the longer sarcomere lengths in ES cell cardiac myocytes compared to myo- cytes from intact hearts. Sarcomere length in foci with densely packed myofibrils arranged into sarcomeres ranged from 2.20-2.01 $\mu \mathrm{m}$ (Fig. 3), and was similar to the sarcomere length in adult mouse cardiac myocytes $(\mathrm{SL}=$ 2.00-2.10 $\mu \mathrm{m}$; Fig. 4).

Another cellular structure which appears during in vivo cardiac myocyte differentiation is the intercalated disc [Bishop et al., 1990; McNutt, 1970]. Electron micrographs of ES cell-derived cardiac myocytes were examined for structural evidence of intercalated discs. Nascent intercalated discs have been observed in contracting ES-D3 cells cultured as embryoid bodies [Doetschman et al., 1985; Robbins et al., 1990]. We also observed nascent intercalated discs in differentiating cardiac myocytes from ES cell cultures (Fig. 5A-D). In addition, we examined intercellular junctions in various sections of ES cell-derived myocytes and identified individual components of the intercalated disc including fascia adherens (Fig. 5A), desmosomes (Fig. 5B,C), and an apparent gap junction (Fig. 5A). In Figure 5A, myofibrils appear to be inserted obliquely into the developing intercalated disc, which is also observed during in vivo cardiac development [Bishop et al., 1990; Manasek, 1970; McNutt, 1970] and in differentiating myocytes grown in oculo [Bishop et al., 1990].

Rhythmic contraction of myocytes within EBs and ES cell foci suggests that intercellular communication occurs in foci of cardiac myocytes within ES cell cultures. Cell-cell communication occurs primarily through the gap junction in heart cells [Barr et al., 1965] and an abundant isoform involved in forming gap junctions throughout cardiac myocyte differentiation is CX-43 [Fromaget et al., 1990; VanKempen et al., 1995]. Thus, we examined whether ES cell derived cardiac myocytes contain gap junctions composed of CX-43. We used a dual monoclonal $\mathrm{Ab}$ immunofluorescence technique described previously [Westfall et al., 1996], and observed punctate CX-43 immunostaining in ES cell-derived cardiac myocytes (Fig. 6B). These cardiac myocytes were identified by the presence of cTnI-specific immunostaining (Fig. 6A) in a focus showing rhythmic contraction prior to fixation. Our results indicate that the punctate immunostaining observed with the CX-43 mAb was present in the same group of cells as the positive TI-1 anti-cTnI mAb immunostaining.

To determine whether these gap junctions were functional, Lucifer yellow was microinjected into individual ES cell cardiac myocytes within a focus of contraction. After injection, the Lucifer yellow spread to adjacent cells within several seconds, which indicates these gap junctions were indeed functional (Fig. 7). Thus, the ultrastructure, CX-43 immunolabeling, and microinjection with Lucifer yellow indicate ES cell-derived cardiac myocytes express intercalated discs with func- 

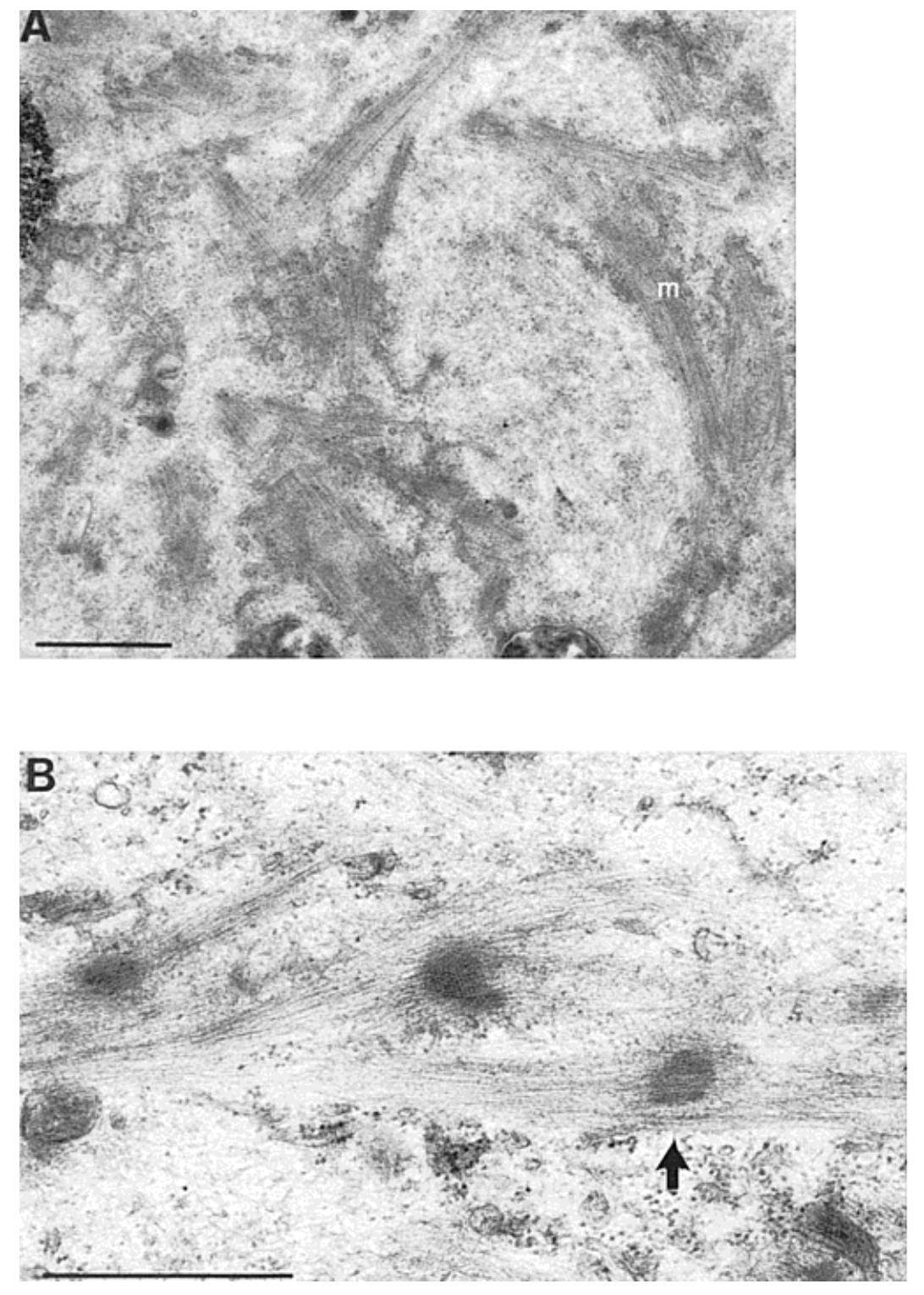

Fig. 2. Representative electron micrographs of cardiac myocytes exhibiting nascent sarcomeres in cultures of ES cells. The white " $\mathrm{m}$ " identifies myofibrils in $\mathbf{A}$, and parallel arrays of myofibrils are evident in B-D. Myofibrillar arrays in B and C also show the nascent Z-line

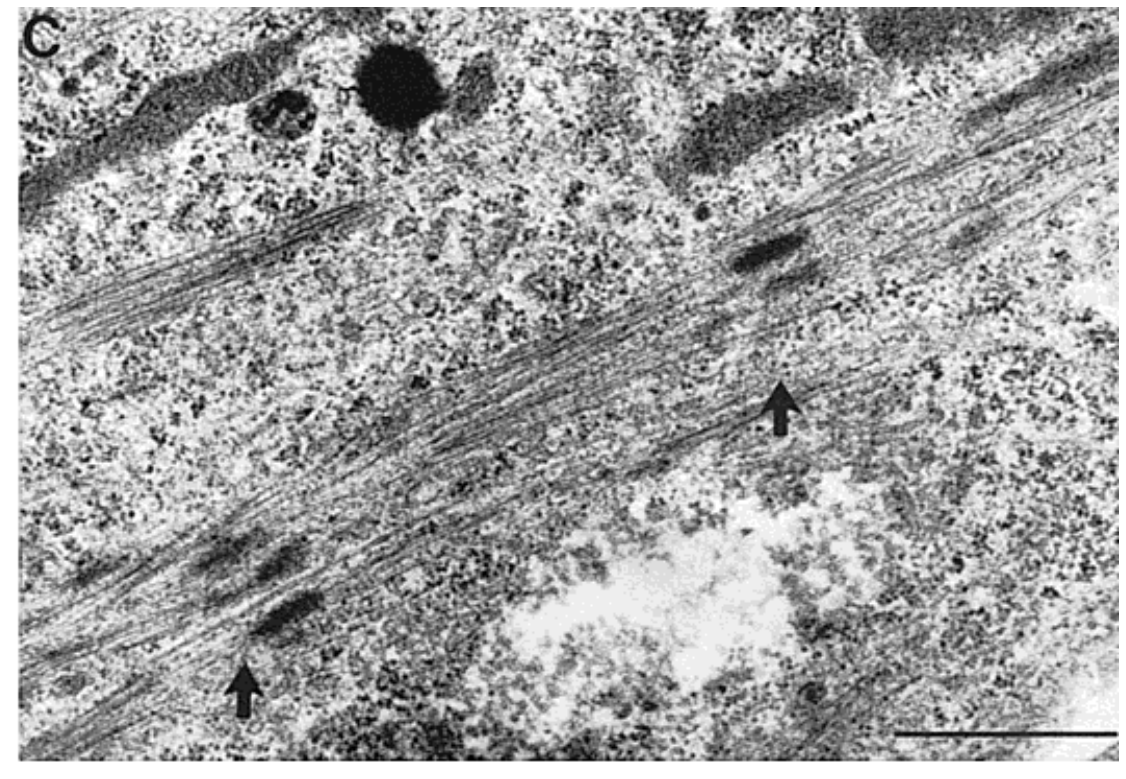

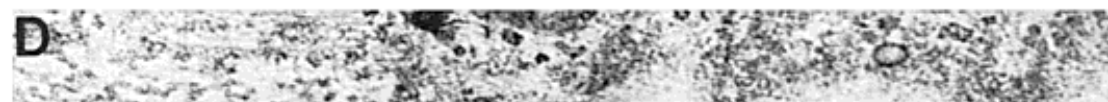

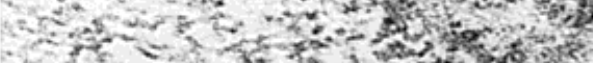

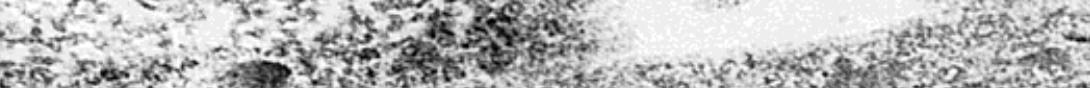

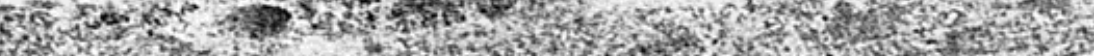

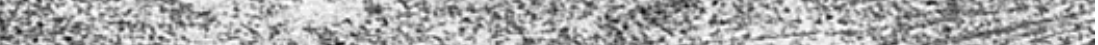

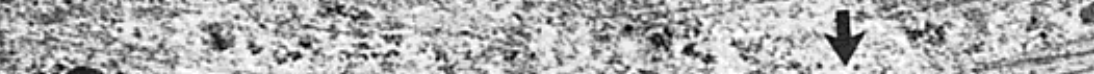

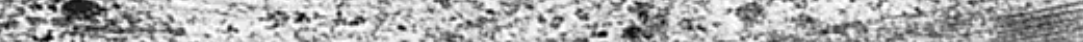

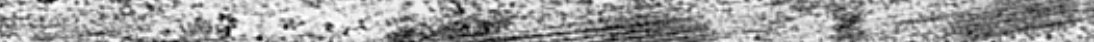

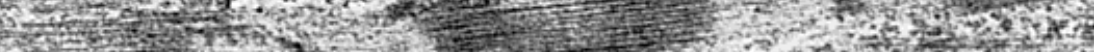
(1)

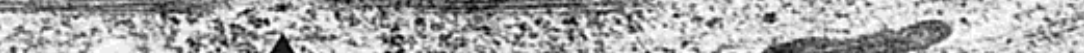

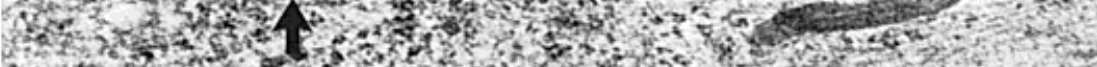

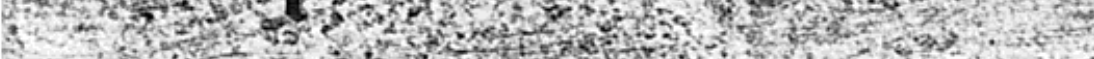

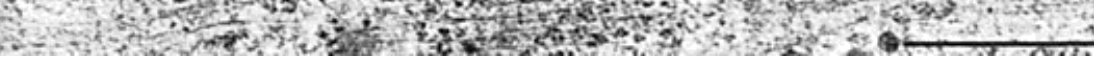

structures (arrows) found in developing cardiac myocytes. Distance

between arrows in $\mathrm{C}$ and $\mathrm{D}$ indicate sarcomere length between adjacent

Z-lines. Bar $=1 \mu \mathrm{m}$ 


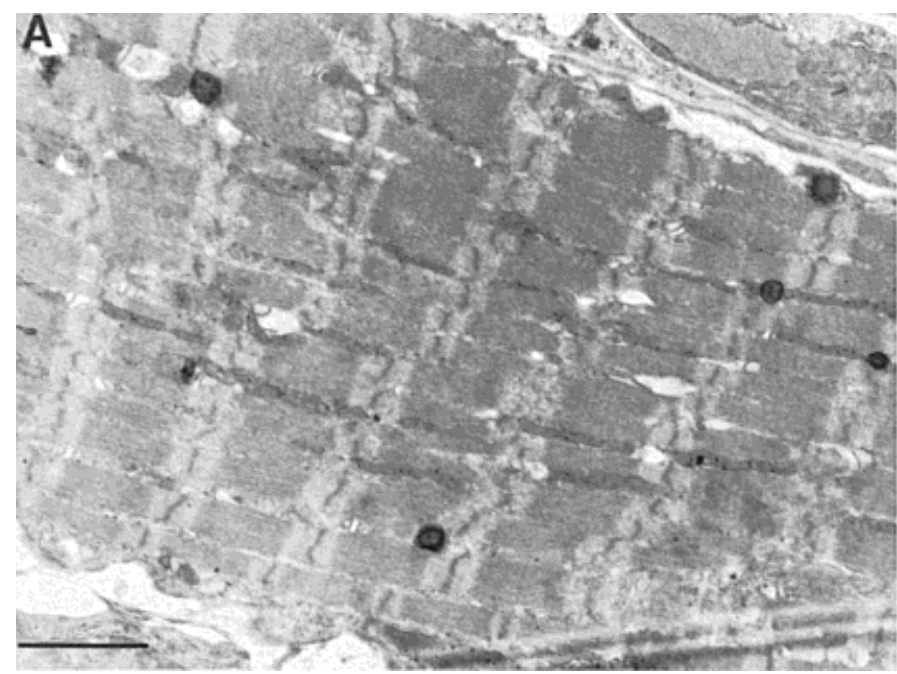

Fig. 3. Electron micrographs of more mature sarcomere structures in ES cell cardiac myocytes $(\mathbf{A}, \mathbf{B})$. A focus which had been contracting for 36 days was microdissected prior to fixation and analysis of sarcomere orientation. The sarcomeres shown in A and B are represen-

tional gap junctions which also develop in ventricular myocytes from intact hearts.

\section{DISCUSSION}

One goal of the present study was to examine in detail the morphometry and cellular ultrastructure of ES cell-derived cardiac myocytes. The detailed examination of cardiac myocytes differentiating in ES cell cultures shows that these cells are mononucleated and rod-shaped (Fig. 1), contain myofibrils arranged in developing sarcomeres (Figs. 2, 3), and exhibit intercalated discs (Fig. 5). This study also provides the first direct evidence that intercalated discs within ES cell-derived cardiac myocytes express the gap junctional subunit, CX-43 (Fig. 6) and that gap junctions within these cardiac myocytes are functionally coupled to adjacent cells (Fig. 7). An additional goal of this study was to determine whether differentiation of ES cell cardiac myocytes at the ultrastructural level follows the pattern observed in cardiac myocytes developing within the heart. Cardiac myocytes in ES-D3 EBs were previously shown to contain sarcomeres and intercalated discs as an indication that these cells exhibited cardiac myocyte characteristics [Doetschman et al., 1985; Robbins et al., 1990]. However, these studies did not determine whether the ultrastructural pattern of development in these cardiac myocytes was similar to cardiac myocyte ultrastructural development in vivo. Results from the present study provide the first strong evidence that the ultrastructural organization in ES cell cardiac myocytes follows a

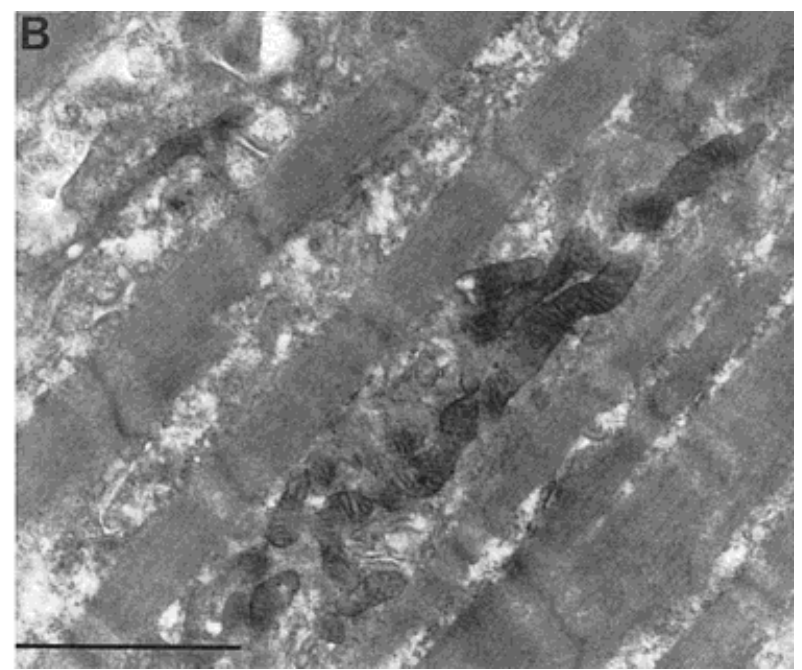

tative of the sarcomere organization observed in this focus. These sarcomeres are similar to the ultrastructure observed in adult myocytes (Fig. 4). Bar $=2 \mu \mathrm{m}$.

pattern of differentiation that is similar to cardiac myocytes in vivo.

The morphometric analysis shows that the cell dimensions of ES cell-derived cardiac myocytes are within the range of dimensions observed in differentiating cardiac myocytes in vivo. Anversa et al. [1980] previously showed that neonatal rodent cardiomyocyte length increases $\sim 2$-fold, diameter increases $\sim 15 \%$, and area increases $\sim 35 \%$, during the first 11 days following birth. The smallest cardiac myocytes observed in the ES cell cultures exhibit length, diameter, and area within this range of dimensions (Table I). Larger myocytes in the ES cell cultures (Table I) have dimensions similar to the adult cardiomyocytes [Korecky and Rakusan, 1978].

Although the cell dimensions of the ES cell cardiac myocytes are consistent with the range of dimensions observed in vivo, it should be noted that a range of cell dimensions was observed within a focus. In addition, the length/diameter (L/D) ratio was consistently higher than values observed in vivo in adult myocytes [Korecky and Rakusan, 1978] and neonates [Anversa et al., 1980]. Heterogeneity in cell dimensions and the higher L/D ratio in ES cell-derived cardiac myocytes within a focus may be expected if factors independent of the differentiation state are affecting cell size. Proliferation of adjacent, nonmyocyte cells within the ES cell culture may influence the size of a contracting focus which, in turn, could affect the size of individual cells within the focus. The availability of cell nutrients to individual cells within a contracting focus may also influence the size of a myocyte, particularly in cultures grown for several weeks. 


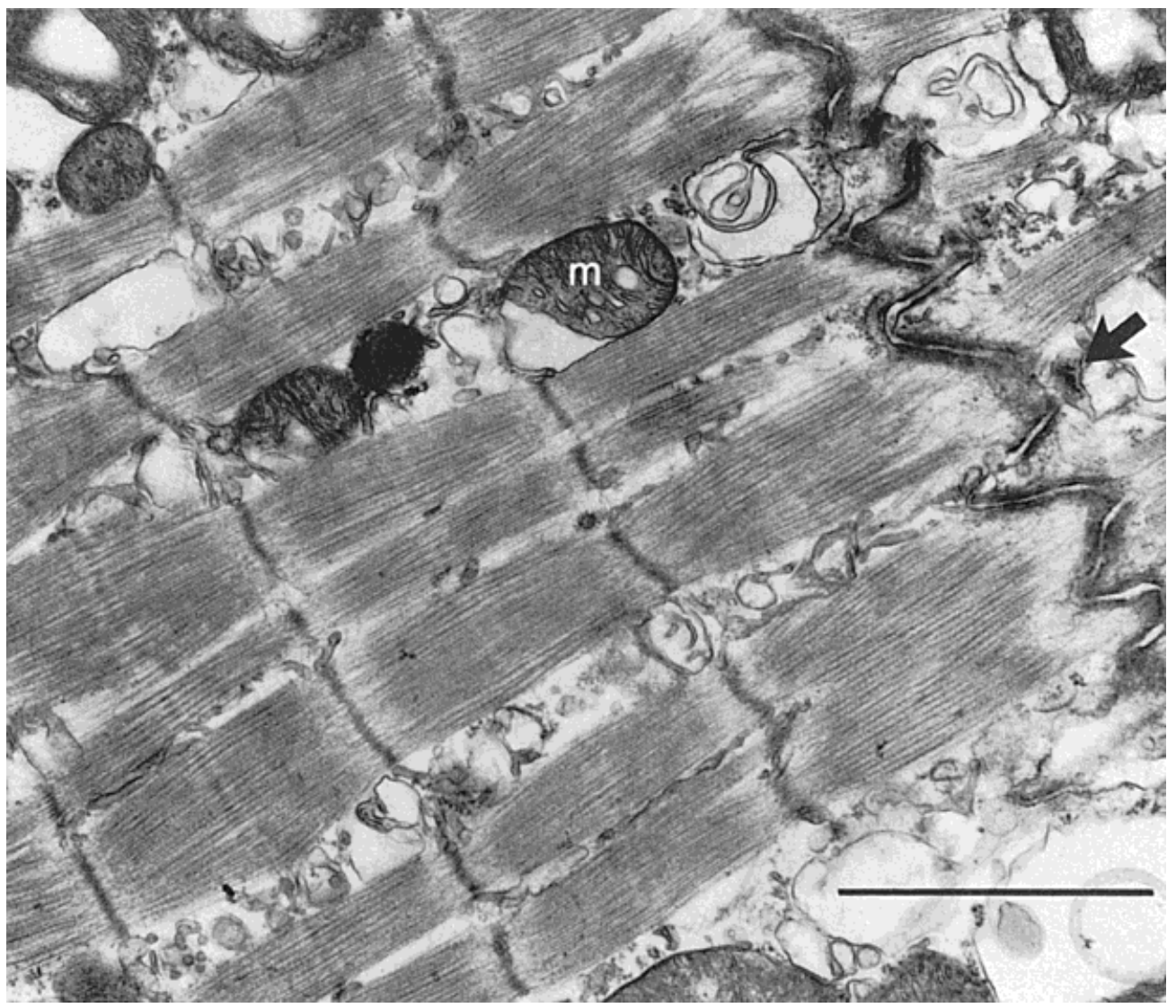

Fig. 4. Representative electron micrograph of sarcomere ultrastructure in adult mouse ventricular cardiac myocyte. The white " $\mathrm{m}$ " indicates mitochondria and the intercalated disc is also apparent on the right side of the picture. The arrow at the intercalated disc designates a gap junction, which electrically couples adjacent myocytes. Bar $=$ $2 \mu \mathrm{m}$.
In addition, Bishop and colleagues [1990] have suggested that myocytes differentiating outside the embryonic/fetal heart may not receive all of the in vivo signals for hypertrophic growth. For example, ES cell cardiac myocytes clearly do not work against the same pressure load observed in vivo. Previous studies [Bishop et al., 1990; Marino et al., 1987; Rohr et al., 1991] supporting this hypothesis have shown that contraction and/or pressure loads cause myocyte hypertrophy and result in increased cell size. Our interpretation of the morphometric results are that many signals which influence cell size in vivo are also present in ES cell cultures. However, at least some factors which lead to cellular hypertrophy in vivo are not present in ES cell cultures.

To further determine whether ES cell cardiac myocyte differentiation is similar to the in vivo development program, we characterized the ultrastructural development of myofibrillar assembly (Figs. 2, 3). Previous studies did not examine the developmental pattern of myofibril organization in ES-D3-derived cardiac myocytes [Doetschman et al., 1985; Robbins et al., 1990]. During in vivo cardiogenesis, myofibrils are initially distributed in sparse, irregular myofibrillar arrays which mature into parallel arrays of myofibrils and ultimately align into densely packed sarcomeres [Chacko, 1976; Manasek, 1970]. In agreement with this pattern, we observed sparse, irregularly organized myofibrils and parallel arrays of myofibrils in foci contracting for short intervals of time (Fig. 2). Densely packed myofibrils organized into well-defined sarcomeres, which are characteristic of adult cardiac myocytes, were observed in the focus which had been contracting for 36 days (Fig. 3). A

Fig. 5. Representative electron micrographs showing developing intercellular junctions in ES cell-derived myocytes. The junctions shown in A-D are similar to those observed during in vivo cardiac differentiation. In $\mathbf{A}$, it appears that myofibrils are obliquely inserted into a developing intercalated disc (upward arrow). An apparent gap junction is shown by the downward arrow. Multiple nascent desmosomes are shown in $\mathbf{B}$ and $\mathbf{C}$. The middle arrow in $\mathbf{C}$ and the arrow in $\mathbf{D}$ also point out oblique insertions of myofibrils into developing intercellular junctions. In each micrograph, the bar $=0.5 \mu \mathrm{m}$ and the white " $\mathrm{m}$ " indicates myofibrils. These specific cultures were incubated for 50 to 65 days and contained foci contracting for 1-53 days. 

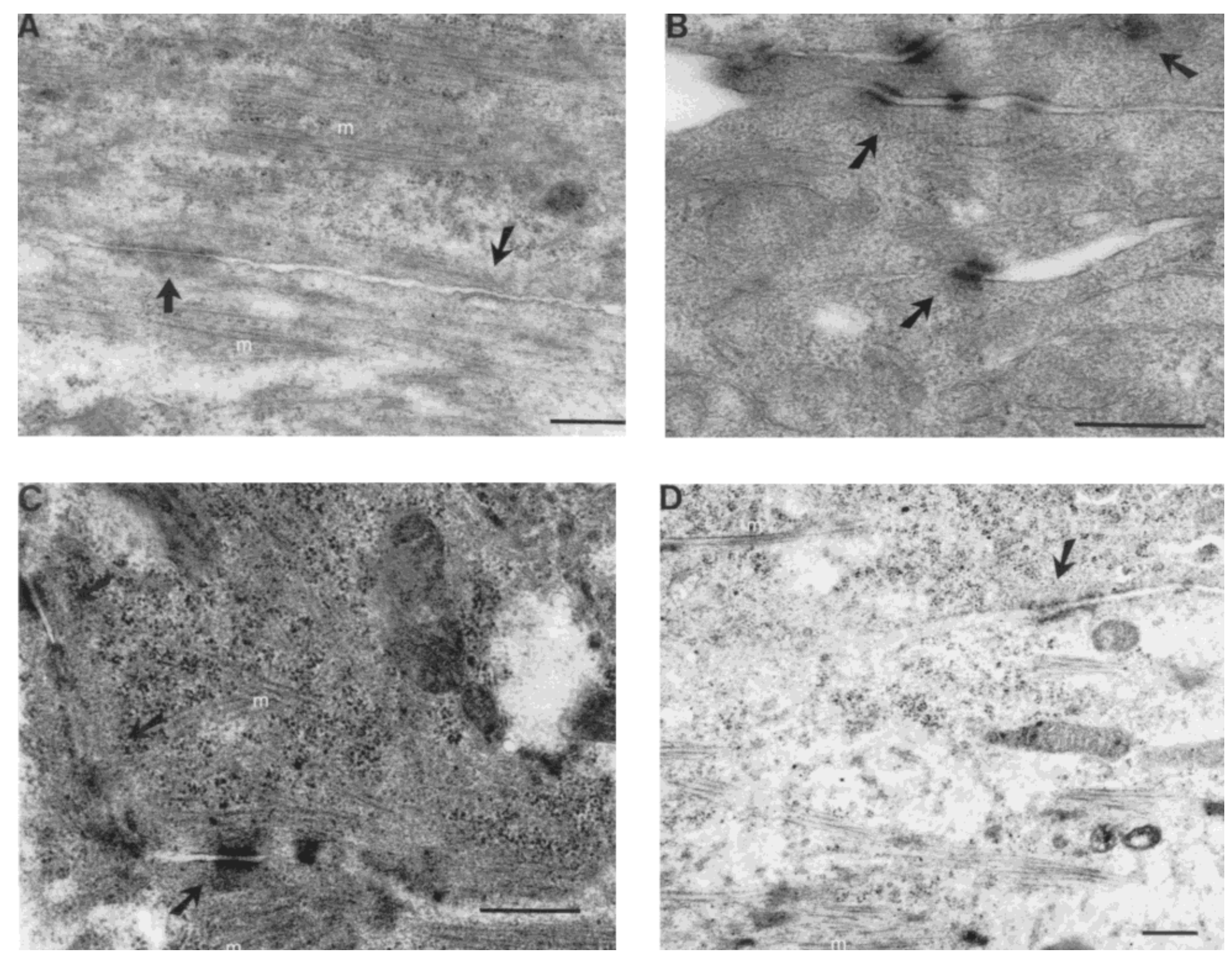


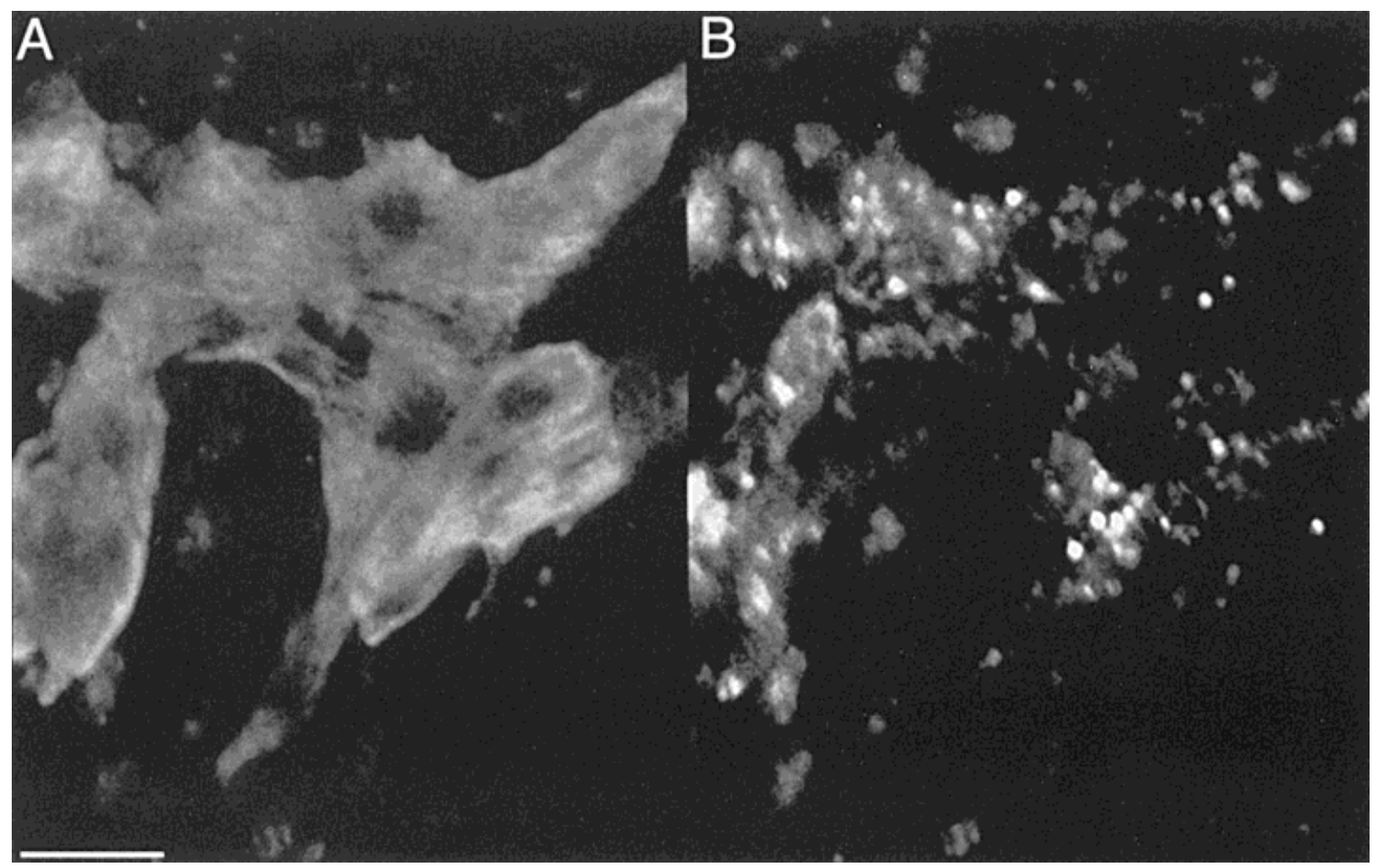

Fig. 6. Indirect immunofluorescence labeling of ES cell-derived cardiac myocytes with monoclonal antibodies recognizing gap junctions and cardiac troponin I (cTnI). Sections were immunolabeled with $\mathrm{TI}-1$ anti-cTnI $\mathrm{Ab}(\mathbf{A})$ and an $\mathrm{Ab}$ which recognizes the CX-43 gap junction protein (B). TI-1 labeling was detected with goat anti-mouse
Ab conjugated to TR (A), while CX-43 labeling was detected with goat anti-mouse conjugated to FITC (B). Confocal microscopy was used to analyze images. These results indicate $\mathrm{CX}-43$ immunostaining is positive within the same area staining positive for $\mathrm{cTnI}$. Bar $=20 \mu \mathrm{m}$.
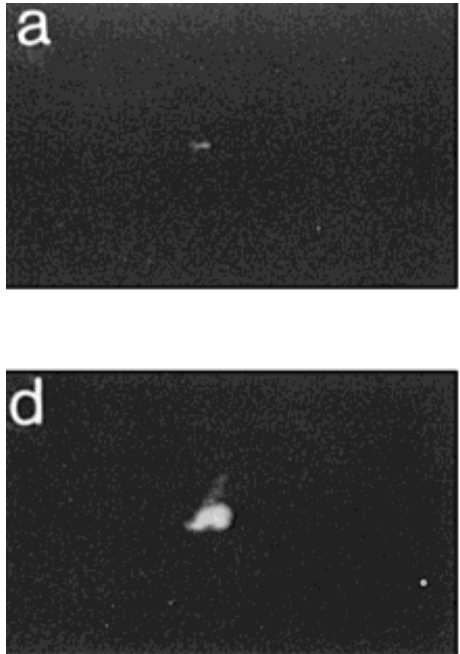
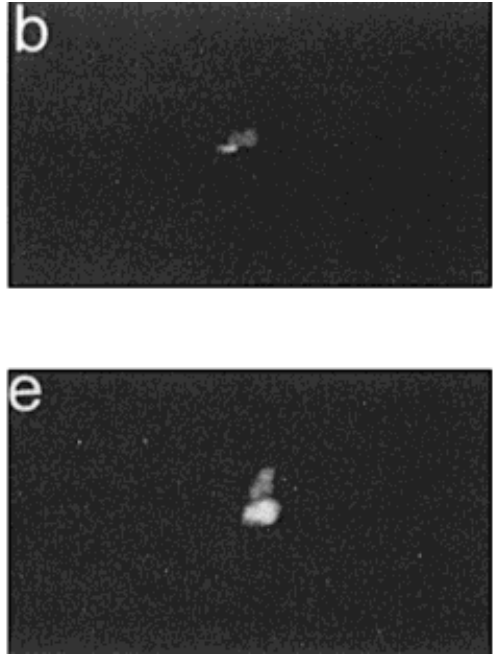
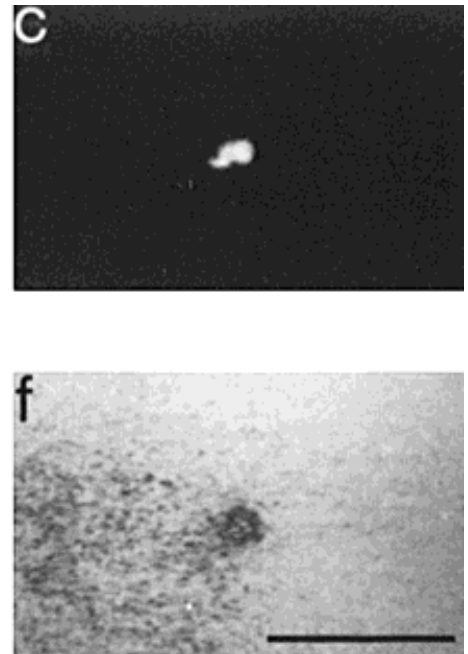

Fig. 7. Microinjection of fluorescent probes into a contracting focus within an ES cell culture. The tip of the pipet is shown in a and the spread of Lucifer yellow signal to adjacent cells is shown at $1 \mathrm{sec}(\mathbf{b}), 2 \mathrm{sec}$ (c), $24 \mathrm{sec}(\mathbf{d})$, and $38 \mathrm{sec}(\mathbf{e})$. The micrograph in $\mathbf{f}$ shows the injection site as detected by the TR-dextran. Bar $=50 \mu \mathrm{m}$.

similar pattern of myofibrillogenesis also was observed in studies using other culture systems to investigate cardiac myocyte differentiation [Bishop et al., 1990; Delcarpio et al., 1991].
Another hallmark of in vivo cardiogenesis is the formation of cellular junctions known as intercalated discs which are composed of fascia adherens, desmosomes, and gap junctions [for review see Forbes and 
Sperelakis, 1985]. The termini of sarcomeres insert into the fascia adherens and transmit force from cell to cell [McNutt, 1970]. Desmosomes provide intercellular adhesion and gap junctions are low resistance sites of intercellular communication [Forbes and Sperelakis, 1985]. Nascent intercalated discs, which are incomplete and with smaller components, are often observed during cardiogenesis [Shibata et al., 1980]. Nascent intercalated discs also have been observed in embryoid bodies [Doetschman et al., 1985; Robbins et al., 1990]. However, individual components of the intercalated disc were not identified in these studies. In the present study, electron micrographs show the fascia adherens, desmosomes, and gap junctions in differentiating ES cell cardiac myocytes (Fig. 5). During the review of this paper, these structures were also observed in G418-selected ES cell cardiac myocytes [Klug et al., 1996].

Gap junctions are a key component of the intercalated disc, which are believed to provide a low resistance pathway coupling adjacent cells such that a functional syncytium is formed in the heart [Spray and Burt, 1990]. Coordinated contraction within a focus provides indirect evidence that ES cell-derived cardiac myocytes contain gap junctions. However, previous investigations have not determined whether functional gap junctions are present. In the current study, a focus of cardiac myocytes identified by the presence of contraction and cTnI-specific immunostaining, also stained positive for CX-43 (Fig. 6), a major gap junction protein found in cardiac myocytes [Darrow et al., 1995; Fromaget et al., 1990; VanKempen et al., 1995]. The spread of Lucifer yellow to adjacent cells following microinjection into ES cell cardiac myocytes (Fig. 7) indicates that these ES myocytes have functionally coupled gap junctions. Lucifer yellow is a dye which penetrates gap junctions but not surface membranes [Stewart, 1981; DeMello et al., 1983]. These results provide the first structural and functional evidence verifying that gap junctions are present in ES cell-derived cardiac myocytes during their differentiation.

In summary, the cell dimensions, pattern of myofibril organization, and genesis of functional intercalated discs in ES cell-derived cardiac myocytes are similar to the pattern observed in vivo. These results, along with previous reports on contractile protein isoform expression [Metzger et al., 1995; Miller-Hance et al., 1993; Muthuchamy et al., 1993; Robbins et al., 1990; Westfall et al., 1996] and contractile function [Metzger et al., 1994], indicate that ES cell cardiac myocyte development holds promise for studying important aspects of cardiogenesis in vitro. For example, factors affecting hyperplastic vs. hypertrophic growth could be analyzed with this model system.

These cells may also provide a source of grafts for infarcted regions of the heart following ischemic insult
[Klug et al., 1996; Soonpaa et al., 1994]. Cardiac myocytes withdraw permanently from the cell cycle during differentiation and so, insults to the myocardium, such as ischemia-mediated necrosis, are irreversible. As a result, investigators are currently examining the possibility of grafting proliferating myocytes into hearts as a means of repairing damage to the myocardium [Klug et al., 1996; Koh et al., 1993a,b; Soonpaa et al., 1994]. Studies with transformed atrial cells [Koh et al., 1993b], transgenic fetal cardiac myocytes [Soonpaa et al., 1994], and skeletal myoblasts [Koh et al., 1993a] indicate grafts become structurally coupled to the endogenous myocardium without negative effects. In addition, Li et al. [1996] have shown that transplanted cell suspensions also grow and form functional myocardium. Foci and/or isolated suspensions of proliferating ES cell cardiac myocytes are another source of myocytes for intracardiac grafting [Klug et al., 1996], and our results indicate grafted ES cell cardiac myocytes have the potential to form functional gap junctions with adjacent myocytes in vitro (Fig. 7). In addition, genetically modified ES cell cardiac myocytes can be generated with relative ease [Klug et al., 1996]. This trait could be exploited to engineer and subsequently graft myocytes into the heart which are able to secrete paracrine factors and thereby influence the surrounding myocardium. These approaches indicate that ES cell cardiac myocytes may be a powerful means for understanding cardiac myocyte function as well as modifying the function of the heart.

\section{ACKNOWLEDGMENTS}

We gratefully acknowledge the generous gift of monoclonal anti-TnI (TI-1) antibody from Dr. Stefano Schiaffino. We also acknowledge the technical assistance of Mr. Ross Johnston and the morphometric analysis provided by the Michigan Diabetes and Training Center. This work was supported by grants from NIH, the American Heart Association, and the American Heart Association of Michigan. J.M.M. is an Established Investigator of the American Heart Association.

\section{REFERENCES}

Anversa, P., Olivetti, G., and Loud, A.V. (1980): Morphometric study of early postnatal development in the left and right ventricular myocardium of the rat. Circ. Res. 46:495-502.

Barr, L., Dewey, M.M., and Berger, W. (1965): Propagation of action potentials and the nexus in cardiac muscle. J. Gen. Physiol. 48:797-823.

Bishop, S.P., Anderson, P.G., and Tucker, D.C. (1990): Morphological development of the rat heart growing in oculo in the absence of hemodynamic work load. Circ. Res. 66:84-102.

Chacko, K.J. (1976): Observations on the ultrastructure of developing myocardium of rat embryos. J. Morphol. 150:681-710.

Darrow, B.J., Laing, J.G., Lampe, P.D., Saffitz, J.E., and Beyer, E.C. 
(1995): Expression of multiple connexins in cultured neonatal rat ventricular myocytes. Circ. Res. 76:381-387.

Delcarpio, J.B., Lanson, N.A., Jr., Field, L.J., and Claycomb, W.C. (1991): Morphological characterization of cardiomyocytes isolated from a transplantable cardiac tumor derived from transgenic mouse atria (AT-1 cells). Circ. Res. 69:1591-1600.

DeMello, W.C., Castillo, M.G., and vanLoon, P. (1983): Intercellular diffusion of lucifer yellow $\mathrm{CH}$ in mammalian cardiac fibers. J. Mol. Cell. Cardiol. 15:637-643.

Deutsch, D.E., Williams, J.A., and Yule, D.I. (1995): Halothane and octanol block $\mathrm{Ca}^{2+}$ oscillations in pancreatic acini by multiple mechanisms. Am. J. Physiol. 269:G779-G788.

Doetschman, T.C., Eistetter, H., Katz, M., Schmidt, W., and Kemler, R. (1985): The in vitro development of blastocyst-derived embryonic stem cell lines: Formation of visceral yolk sac, blood islands and myocardium. J. Embryol. Exp. Morphol. 87:27-45.

Forbes, M.S., and Sperelakis, N. (1985): Intercalated discs of mammalian heart: A review of structure and function. Tissue Cell 17:605-648.

Fromaget, C., El Aoumari, A., Dupont, E., Briand, J.P., and Gros, D. (1990): Changes in the expression of connexin 43, a cardiac gap junctional protein, during mouse heart development. J. Mol. Cell. Cardiol. 22:1245-1258.

Klug, M.G., Soonpaa, M.H., Koh, G.Y., and Field, L.J. (1996): Genetically selected cardiomyocytes from differentiating embryonic stem cells form stable intracardiac grafts. J. Clin. Invest. 98:216-224.

Koh, G.Y., Klug, M.G., Soonpaa, M.H., and Field, L.J. (1993a): Differentiation and long-term survival of $\mathrm{C} 2 \mathrm{C} 12$ myoblast grafts in heart. J. Clin. Invest. 92:1548-1554.

Koh, G.Y., Soonpaa, M.H., Klug, M.G., and Field, L.J. (1993b): Long-term survival of AT-1 cardiomyocyte grafts in syngeneic myocardium. Am. J. Physiol. 264:H1727-1733.

Korecky, B., and Rakusan, K. (1978): Normal and hypertrophic growth of the rat heart: Changes in cell dimensions and number. Am. J. Physiol. 234:H123-H128.

Li, R.K., Mickle, D.A.G., Weisel, R.D., Zhang, J., and Mohabeer, M.K. (1996): In vivo survival and function of transplanted rat cardiomyocytes. Circ. Res. 78:283-288.

Mahdavi, V., Izumo, S., and Nadal-Ginard, B. (1987): Developmental and hormonal regulation of sarcomeric myosin heavy chain gene family. Circ. Res. 60:804-814.

Manasek, F.J. (1970): Histogenesis of the embryonic myocardium. Am. J. Cardiol. 25:149-168.

Marino, T.A., Kuseryk, L., and Lauva, I.K. (1987): Role of contraction in the structure and growth of neonatal rat cardiomyocytes. Am. J. Physiol. 253:H1391-H1399.

McNutt, S. (1970): Ultrastructure of intercellular junctions in adult and developing cardiac muscle. Am. J. Cardiol. 25:169-183.

Metzger, J.M., Lin, W., and Samuleson, L.C. (1994): Transition in cardiac contractile sensitivity to calcium during the in vitro differentiation of mouse embryonic stem cells. J. Cell Biol. 126:701-711.

Metzger, J.M., Lin, W., Johnston, R.A., Westfall, M.V., and Samuelson, L.C. (1995): Myosin heavy chain expression in contracting myocytes isolated during embryonic stem cell cardiogenesis. Circ. Res. 76:710-719.

Miller-Hance, W.C., LaCorbiere, M., Fuller, S., Evans, S., Lyons, G., Schmidt, C., Robbins, J., and Chien, K. (1993): In vitro chamber specification during embryonic stem cell cardiogenesis. J. Biol. Chem. 268:25244-25252.

Muthuchamy, M., Pajak, L., Howles, P., Doetschman, T., and Wieczorek, D. (1993): Developmental analysis of tropomyosin gene expression in embryonic stem cells and mouse embryos. Mol. Cell. Biol. 13:3311-3323.

Robbins, J., Gulick, J., Sanchez, A., Howles, P., and Doetschman, T. (1990): Mouse embryonic stem cells express the cardiac myosin heavy chain genes during development in vitro. J. Biol. Chem. 265:11905-11909.

Robbins, J., Doetschman, T., Jones, W.K., and Sanchez, A. (1992): Embryonic stem cells as a model for cardiogenesis. Trends Cardiovasc. Med. 2:44-50.

Rohr, S., Scholly, D.M., Kleber, A.G. (1991): Patterned growth of neonatal rat heart cells in culture. Circ. Res. 68:114-130.

Saggin, L., Gorza, L., Ausoni, S., and Schiaffino, S. (1989): Troponin I switching in the developing heart. J. Biol. Chem. 264:1629916302.

Severs, N.J. (1989): Constituent cells of the heart and isolated cell models in cardiovascular research. In Piper, H.M., and Isenberg, G. (eds): "Isolated Adult Cardiomyocytes." Boca Raton, FL: CRC Press, Inc. pp. 3-41.

Shibata, Y., Nakata, K., and Page, E. (1980): Ultrastructural changes during development of gap junctions in rabbit left ventricular myocardial cells. J. Ultrastruct. Res. 71:258-271.

Soonpaa, M.H., Koh, G.Y., Klug, M.G., and Field, L.J. (1994): Formation of nascent intercalated disks between grafted fetal cardiomyocytes and host myocardium. Science 264:98-101.

Spray, D.C., and Burt, J.M. (1990): Structure-activity relations of the cardiac gap junction channel. Am. J. Physiol. 258:C195-C205.

Stewart, W.W. (1981): Lucifer dyes: Highly fluorescent dyes for biological tracing. Nature 292:17-21.

VanKempen, M.J.A., TenVelde, I., Wessels, A., Oosthoek, P.W., Gros, D., Jongsma, H.J., Moorman, A.F.M., and Lamers, W.H. (1995): Differential connexin distribution accommodates cardiac function in different species. Microsc. Res. Tech. 31:420-436.

Westfall, M.V., Samuelson, L.C., and Metzger, J.M. (1996): Troponin I isoform expression is developmentally regulated in differentiating embryonic stem cell-derived cardiac myocytes. Dev. Dyn. 206:24-38. 